adsorption. $B$ and $C$ iteration parameters for $C, N$, and $O$ atoms were taken from ref 95 . Extended Hückel parameters for all atoms used are listed in Table XII.

(95) McGlyn, S. P.; Vanquickenborne, L. G.; Kinoshita, M.; Carroll D. G. Introduction to Applied Quantum Chemistry; Holt, Rinehart and Winston: New York, 1964; pp 423-431.
All the results listed in this paper are from the singleface adsorption calculations, using the three-layer slab model. The geometrical parameters for coadsorption are as follows: $\mathrm{C}-\mathrm{O}, 1.15 ; \mathrm{N}-\mathrm{O}, 1.15$; $\mathrm{Rh}-\mathrm{Rh}, 2.6893$; $\mathrm{Rh}-\mathrm{C}$, 1.90; $\mathrm{Rh}-\mathrm{N}, 1.90$; and $\mathrm{Rh}-\mathrm{O}, 2.05$ (all values in $\AA$ ). The $k$-point sets varied from 49 to 12 points in order to keep the total number of states for each geometry similar.

\title{
Determination of the Electrostatic Potential of Positively Charged Monolayers at the Air/Water Interface by Means of Fluorometric Titration of 4-Heptadecyl-7-hydroxycoumarin
}

\author{
Jordan G. Petrov* \\ Department of Physical Chemistry, University of Sofia, 1, Anton Ivanov Ave., \\ 1126 Sofia, Bulgaria \\ Dietmar Möbius \\ Max-Planck-Institut für biophysikalische Chemie, Postfach 2841, D-3400 Göttingen, FRG \\ Received June 14, 1989. In Final Form: October 16, 1989

\begin{abstract}
The electrostatic potentials $\psi_{0}$ of insoluble monolayer mixtures of a neutral and a positively charged component spread onto an aqueous solution of $0.1 \mathrm{M} \mathrm{NaCl}$ are determined by means of fluorometric titration of embedded 4-heptadecyl-7-hydroxycoumarin. Methyl arachidate and dimethyldioctadecylammonium bromide are used, and the surface charge density $\sigma_{0}$ is varied through their molar ratio. The $\psi_{0} / \sigma_{0}$ dependence thus obtained is compared to experimental results reported in the literature for other systems as well as to the Gouy-Chapman theoretical relationship.
\end{abstract}

\section{Introduction}

The experimental determination of the electrostatic potential $\psi_{0}$ between a nonconducting phase and an aqueous electrolyte solution is a complicated problem. At the same time, its successful solution is of substantial interest due to the important role this parameter plays in the colloid stability, wetting, biomembrane functioning, cell adhesion, etc.

The development of the theory of thin liquid film stability has enabled the determination of $\psi_{0}$ from measurements of equilibrium film thicknesses. ${ }^{1}$ In this way, $\psi_{0}$ values for the air/water interface have been obtained by investigation of foam films stabilized with anionic and cationic surfactants. ${ }^{1-4}$ However, due to restriction of the theoretical calculation of the electrostatic disjoining pressure, reliable data on $\psi_{0}$ can be obtained so far at low ionic strengths only.

In the studies of the electrostatic properties of biomembranes and micelles, $\psi_{0}$ is determined by means of various spectroscopic techniques. Those based on molecu-

* To whom correspondance should be addressed.

(1) Derjaguin, B. V.; Titijevskaya, A. Kolloidn. Zh. 1953, 15, 416. 24.

(2) Scheludko, A.; Exerowa, D. Kolloid-Z. 1959, 165, 148; 1960, 168,

(3) Exerowa, D. Kolloid-Z. 1969, 232, 703

(4) Exerowa, D. Dr. Sci. Thesis, Bulg. Acad. Sci., Sofia, 1987. lar spin probes ${ }^{5}$ and interfacial $\mathrm{pH}$ indicators ${ }^{6}$ are most often employed. In the latter case, $\psi_{0}$ is evaluated from the difference in the $\mathrm{p} K$ values of the indicator at the interface and in the bulk, both being determined by following the absorption or fluorescence changes with $\mathrm{pH}$. (In later studies, $\psi_{0}$ is related to the difference between $\mathrm{p} K_{\mathrm{i}}$ in a neutral and a charged interface. ${ }^{8}$ )

Fromherz et al., ${ }^{7-10}$ as well as some Australian colleagues, ${ }^{11-14}$ have contributed substantially to the progress and recent intensive application of this method. These studies deal with neutral and charged micelles and insoluble monolayers transferred onto a hydrophobic solid substrate by means of the Langmuir-Blodgett technique.

In the present investigation, the electrostatic poten-

(5) Castle, J. D.; Hubbell, W. L. Biochemistry 1976, 15, 4818.

(6) Haynes, D. H. J. Membr. Biol. 1974, 17, 341 .

(7) Fromherz, P. Biochim. Biophys. Acta 1973, 323, 326.

(8) Fromherz, P,; Masters, B. Biochim. Biophys. Acta 1974, 356, 270

(9) Fernandez, M. S.; Fromherz, P. J. Phys. Chem. 1977, 81, 1755.

(10) Fromherz, P.; Kotulla, R. Ber. Bunsen-Ges. Phys. Chem. 1984, 88,1106 .

(11) Lovelock, B.; Grieser, F.; Healy, T. W. J. Phys. Chem. 1985, 89, 501.

(12) Lovelock, B.; Grieser, F.; Healy, T. W. Langmuir 1986, $2,443$.

(13) Drummond, C. J.; Warr, G. G.; Grieser, F.; Ninham, B. W.; Evans,

D. F. J. Phys. Chem. 1985, 89, 2103.

(14) Drummond, C. J.; Grieser, F. Photochem. Photobiol. 1987, 45, 19. 


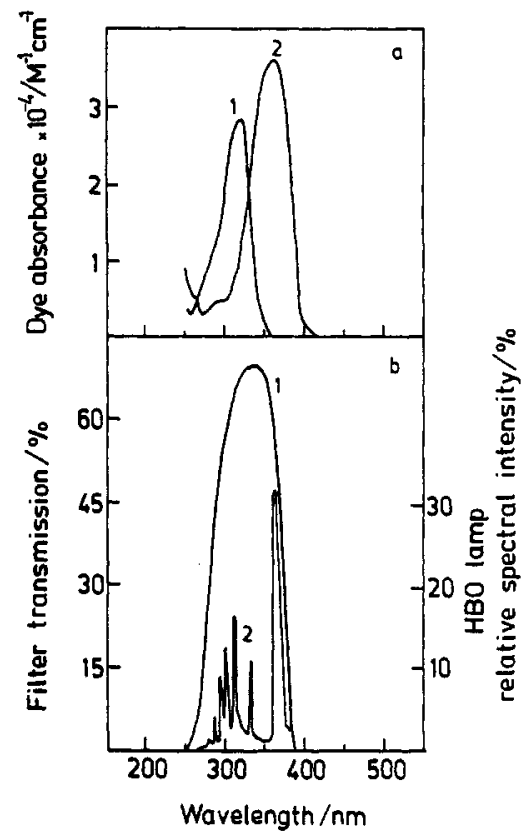

Figure 1. (a) Absorption spectra of the neutral (1) and anionic (2) forms of 4-heptadecyl-7-hydroxycoumarin. (b) UV-11 filter transmission spectrum (1) and relative spectral intensity of the excitation HBO 100 lamp.

tials of monolayers spread on the air/water interface are determined. An experimental technique developed earlier ${ }^{16}$ for recording fluorescence spectra of spread monolayers is applied. Mixtures containing neutral and positively charged components, methyl arachidate (MA) and dimethyldioctadecylammonium bromide (DOMA), are used to vary the surface charge density $\sigma_{0}$. The fluorescence spectra of 4-heptadecyl-7-hydroxycoumarin (HHC) embedded in these matrices are recorded at different $\mathrm{pH}$ values of the subsolution. From the titration curves, the $\mathrm{p} K_{\mathrm{i}}^{0}$ and $\mathrm{p} K_{\mathrm{i}}$ values (for the neutral and the charged monolayers, respectively) are obtained and $\psi_{0}$ is calculated from their difference.

This titration requires the use of buffer solutions due to the large air/monolayer contact area. For this reason, aqueous subsolutions of relatively high $\mathrm{NaCl}$ concentration are applied so the double electric layer can be regarded as being built up of 1:1 electrolyte only.

\section{Method and Materials}

The experimental setup and procedure for recording fluorescence spectra of dyes embedded into insoluble monolayers at the liquid/gas interface are described in ref 15 . In the present study, the excitation monochromator is replaced by a UV-11 filter providing a more intensive illumination and better signal/ noise ratio. However, due to the broad transmission region of the filter, a simultaneous excitation of the HHC anionic form (by the bands at 366 and $334 \mathrm{~nm}$ ) and its neutral form (at 289, $297,302,312$, and $334 \mathrm{~nm}$ ) is observed - see Figure 1. On the one hand, this type of excitation leads to more complicated spectral patterns since the spectra of the two forms are superimposed. On the other hand, the neutral form is invisible with monochromatic excitation at $366 \mathrm{~nm}$ since it does not absorb at this wavelength (Figure 1a). In this way, it becomes possible to follow the changes in the spectra of both forms related to molecular association, interactions with the monolayer molecules and the subsolution components, influence of the interfacial electrostatic potential, and other effects.

As in the previous study, the fluorescence spectra are obtained as differences between the HHC spectra in the monolayer and those of the pure liquid surface. ${ }^{15}$ During the measurement,

(15) Petrov, J. G.; Möbius, D. Langmuir 1989, 5, 523.

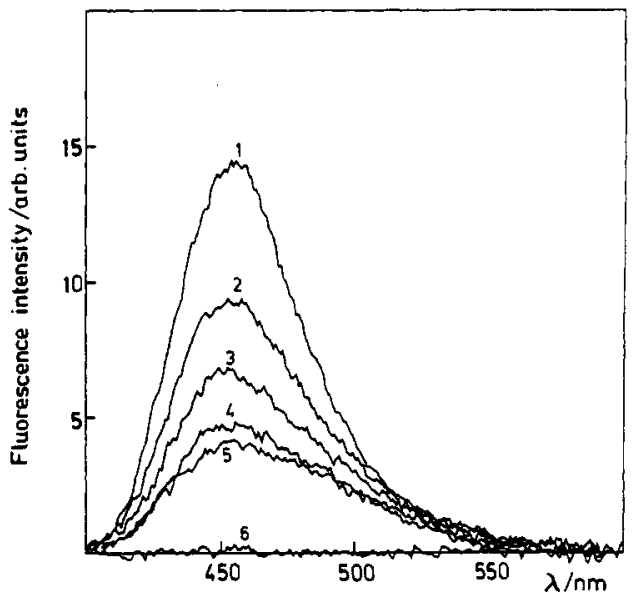

Figure 2. Fluorescence spectra of HHC in methyl arachidate monolayers on $1 \times 10^{-2} \mathrm{M}$ phosphate buffer. Molar ratio of the dye was $1 / 400: 1, \mathrm{pH} 11.9 ; 2, \mathrm{pH} 8.1 ; 3, \mathrm{pH} 7.5 ; 4, \mathrm{pH} 6.9$; $5, \mathrm{pH} 5.5 ; 6$, pure MA without HHC.

the monolayer is maintained at a constant surface pressure of $30 \mathrm{dyn} / \mathrm{cm}$. The relative change in the monolayer area is controlled as a measure of its stability. The solution in the Langmuir trough is maintained at a constant temperature of $20.5 \pm$ $0.2^{\circ} \mathrm{C}$.

Methyl arachidate for chromatographic purposes (Merck) and dimethyldioctadecylammonium bromide produced by Kodak are used as $1 \times 10^{-3} \mathrm{M}$ solutions in chloroform; these are mixed at predetermined molar rations. 4-Heptadecyl-7-hydroxycoumarin ( $\mathrm{mp} 92-93^{\circ} \mathrm{C}$ ) is synthesized according to Sondermann. ${ }^{16}$

The aqueous subsolutions are prepared with water from a Milli-Q system. $\mathrm{pH}$ is adjusted by means of phosphate buffer ( $\mathrm{KH}_{2} \mathrm{PO}_{4}$ and $\mathrm{Na}_{2} \mathrm{HPO}_{4}$, Merck, AR purity grade), $1 \mathrm{M} \mathrm{HCl}$, and $1 \mathrm{M} \mathrm{NaOH}$, both ultrapure. The ionic strength of the solutions is adjusted with ultrapure $\mathrm{NaCl}$.

The $\mathrm{pH}$ of the aqueous subsolutions is kept constant within $0.1 \mathrm{pH}$ unit; control measurements after the completion of the experiment have been carried out by taking samples from the trough.

\section{Results}

Figure 2 shows fluorescence spectra of $\mathrm{HHC}$ in a neutral methyl arachidate monolayer at different $\mathrm{pH}$ values. Curve 6 is a blank control obtained at $\mathrm{pH} 11.9$ as the difference between the spectrum of HHC-free MA monolayer and that of the pure liquid surface. The aqueous subsolutions are chosen to be the same as in our previous investigation of this matrix ${ }^{15}$ (a phosphate buffer with almost constant ionic strength of $1 \times 10^{-2} \mathrm{M}$ ) in order to compare the two excitation techniques. At the same time, the $\mathrm{p} K_{\mathrm{i}}^{0}$ value obtained from these spectra can be used as a reference for the determination of $\psi_{0}$ on $0.1 \mathrm{M} \mathrm{NaCl}$ subsolution. According to the results of ref 8 , the $\mathrm{p} K_{\mathrm{i}}^{0}$ of $\mathrm{HHC}$ in methyl stearate monolayers does not depend on the ionic strength.

In Figure 3 , the $I_{\lambda_{\text {ar }}} / \mathrm{pH}$ dependence for the MA matrix is presented by curve 1 . It has the characteristic $S$ shape obtained also by titration of the coumarin anion in ref 15 but does not have a well-defined plateau in the acidic range. ${ }^{24}$ This trend restrains the calculation of the degree of dissociation $\alpha$ of $\mathrm{HHC}$ and hence of $\mathrm{p} K_{i}^{0}$ since

$$
\alpha=\frac{I-I_{\mathrm{HA}}}{I_{\mathrm{A}}-I_{\mathrm{HA}}}
$$

(16) Möbius, D.; Bücher, H.; Kuhn, H.; Sondermann, J. Ber. Bunsen-Ges. Phys. Chem. 1969, 73, 835. 


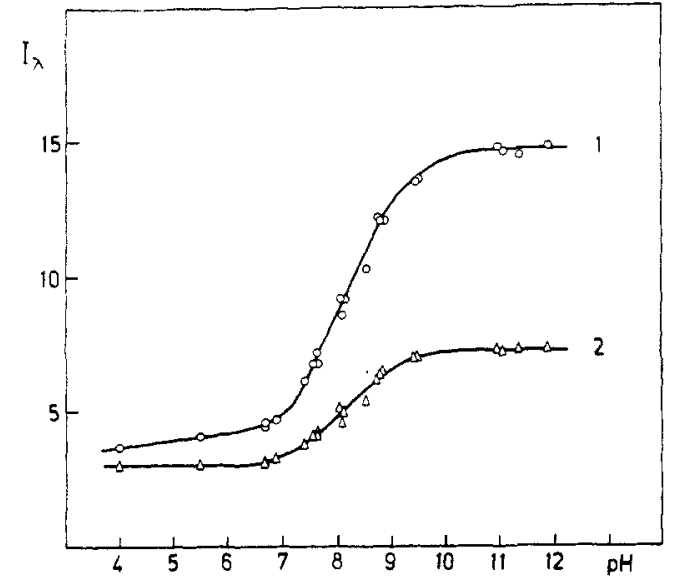

Figure 3. Fluorescence intensity of HHC in MA monolayer on $1 \times 10^{-2} \mathrm{M}$ phosphate buffer at $\lambda_{\max }=455 \mathrm{~nm}$ (1) and at $\lambda$ $=485 \mathrm{~nm}(2)$ versus $\mathrm{pH}$. $\mathrm{HHC} / \mathrm{MA}=1 / 400$.

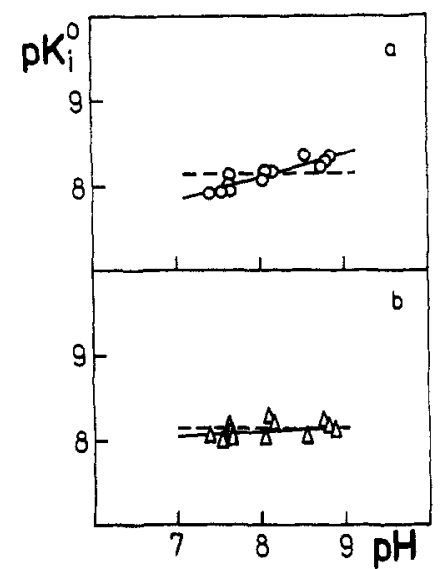

Figure 4. $\mathrm{pH}$ dependence of $\mathrm{p} K_{\mathrm{i}}{ }^{0}$ calculated from eq 1 and 2 and the data shown in Figure 3 : (a) $\lambda=455 \mathrm{~nm}$; (b) $\lambda=485$ nm.

$$
\mathrm{p} K_{\mathrm{i}}^{0}=\mathrm{pH}-\log \frac{\alpha}{1-\alpha}
$$

$I_{\mathrm{A}}$ and $I_{\mathrm{HA}}$ are the fluorescence intensities of the anionic and neutral form of $\mathrm{HHC}$, respectively. $I_{\mathrm{A}}$ is determined from the plateau in the alkaline region while $I_{\mathrm{HA}}$ is defined by a corresponding plateau in the acidic region.

The above complication does not represent an exceptional case in the spectroscopic determination of ionization constants; it can be avoided by following the $\mathrm{pH}$ dependence of the fluorescence intensities at another wavelength. ${ }^{17}$ Curve 2 in Figure 3 is obtained in this manner at $\lambda=485 \mathrm{~nm}$.

According to ref 17 , the absence of a plateau in the acidic region should lead to a $\mathrm{pH}$ dependence of the $\mathrm{p} K_{\mathrm{i}}^{0}$ values determined at $\lambda_{\max }$. This fact is illustrated by Figure 4, where the effect of the change in the analytical wavelength is also shown. In order to diminish the strongly increasing error at high and low $\alpha$ values, $\mathrm{pK}_{\mathrm{i}}{ }^{0}$ is calculated within a narrow $\mathrm{pH}$ range around $\alpha=$ 0.5 . At $455 \mathrm{~nm}$, the minimum fluorescence intensity at $\mathrm{pH} 4.0$ is taken for $I_{\mathrm{HA}}$. The mean $\mathrm{p} K_{\mathrm{i}}{ }^{\circ}$ values are marked with a dashed line; they coincide with each other and are equal to the $\mathrm{p} K_{\mathrm{i}}^{0}$ value of the same system obtained by titration of the anion form alone in ref 15 .

Spectra of HHC in a mixed DOMA/MA monolayer at molar ratio 1:10 are presented in Figure 5. For this and

(17) Albert, A.; Serjeant, E. P. Determination of Ionization Constants; Chapmen and Hall: London, 1971; p 56-59.

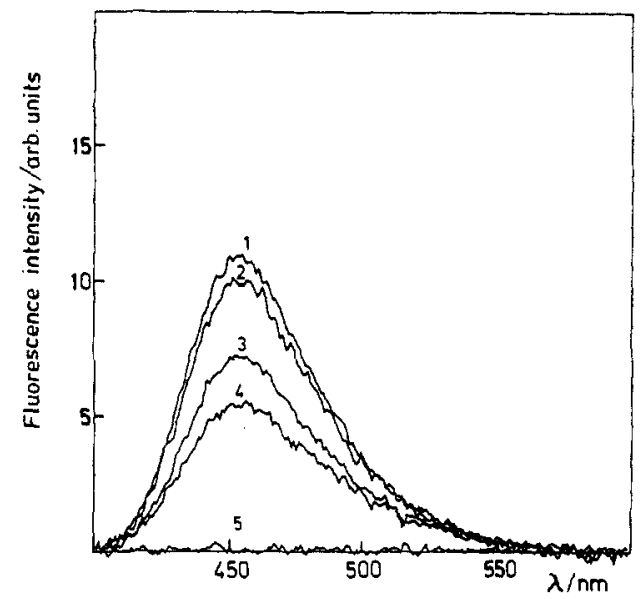

Figure 5. Fluorescence spectra of HHC in 1/10 DOMA/MA mixtures on $0.1 \mathrm{M} \mathrm{NaCl}$. Molar ratio of HHC to (DOMA + $\mathrm{MA})=1 / 400: 1, \mathrm{pH} 9.0 ; 2, \mathrm{pH} 7.5 ; 3, \mathrm{pH} 6.9 ; 4, \mathrm{pH} 5.5 ; 5$, pure matrix without HHC.

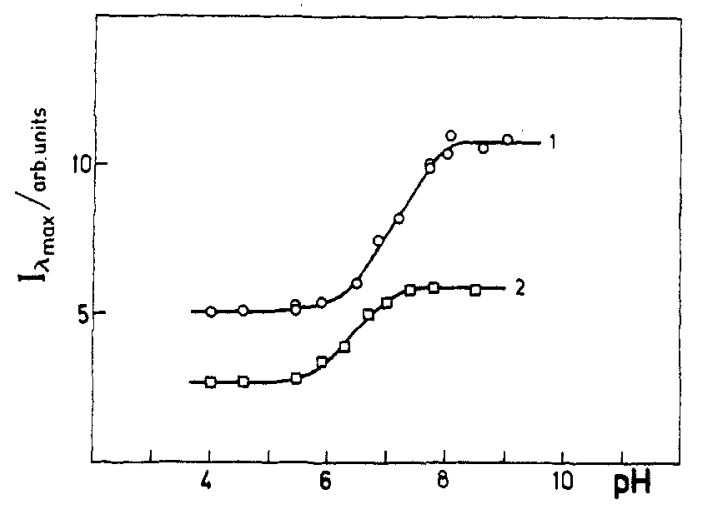

Figure 6. Fluorometric titration curves of $\mathrm{HHC}$ in 1/10 (1) and $1 / 2$ (2) DOMA/MA mixtures on $0.1 \mathrm{M} \mathrm{NaCl}$. Molar ratio of $\mathrm{HHC} /$ matrix $=1 / 400$.

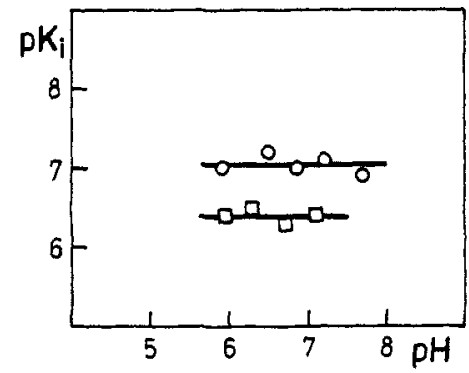

Figure 7. $\mathrm{pH}$ dependence of $\mathrm{p} K_{\mathrm{i}}$ for DOMA/MA mixtures with molar ratios of $1 / 10$ (circles) and $1 / 2$ (squares). Subsolution was $0.1 \mathrm{M} \mathrm{NaCl}$.

all other charged matrices, the aqueous subsolutions contain $1 \times 10^{-4} \mathrm{M}$ phosphate buffer and $0.1 \mathrm{M} \mathrm{NaCl}$. The buffer concentration is lower by 3 orders of magnitude than that of $\mathrm{NaCl}$, so if not very strong specific adsorption of phosphate anions takes place in the double electric layer, the latter could be considered as built up of 1:1 electrolyte.

Titration curves taken at $\lambda_{\max }$ for two DOMA/MA molar ratios are presented in Figure 6 . For both systems, the $I_{\lambda} \quad$ values at $\mathrm{pH} 4.5$ and 4.0 coincide within the limits of the noise of the signal, thus defining a plateau in the acidic range. For this reason, the above mentioned problems in the calculation of $\alpha$ and $\mathrm{p} K_{\mathrm{i}}{ }^{0}$ are not encountered in these cases.

Comparison of Figures 2 and 5 suggests that the surface charges of DOMA do not cause a noticeable change in the position of the maximum and the shape of the 


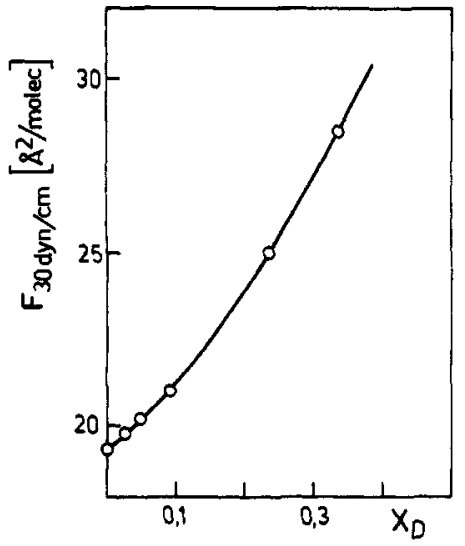

Figure 8. Mean area per molecule at $30 \mathrm{dyn} / \mathrm{cm}$ in DOMA/ MA mixed monolayers versus the molar part of DOMA in the mixture.

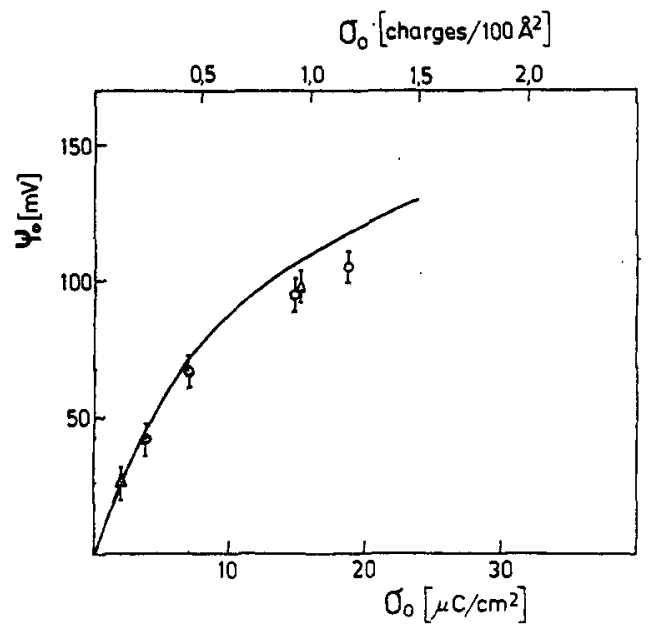

Figure 9. Dependence of the interfacial potential $\psi_{0}$ on the interfacial charge density $\sigma_{0}$. Excitation with UV-11 filter (circles) and monochromator at $366 \mathrm{~nm}$ (triangles). The solid line is drown according to the Gouy-Chapman equation. ${ }^{22}$

HHC spectra. However, the different values of the plateau in the acidic and alkaline regions presented in Figure 6 show that the fluorescence intensities of the anionic and neutral forms decrease with the rise of the surface charge. We are not in a position to comment on this effect now, but it seems that it does not compromise the calculated $\mathrm{p} K_{\mathrm{i}}$ values - they remain $\mathrm{pH}$-independent, as shown in Figure 7.

From $\mathrm{p} K_{\mathrm{i}}^{0}=8.2$ for the neutral MA matrix and the $\mathrm{p} K_{\mathrm{i}}$ values for the charged mixed DOMA/MA monolayers, the electric potential at the interface studied can be calculated:

$$
\psi_{0}=\frac{2.303 R T}{F}\left(\mathrm{p} K_{\mathrm{i}}^{0}-\mathrm{p} K_{\mathrm{i}}\right)
$$

Here $R$ is the gas constant, $T$ is the absolute temperature, and $F$ is the Faraday equivalent.

The surface charge density $\sigma_{0}$ (in charges per $100 \AA^{2}$ ) can be calculated by the formula

$$
\sigma_{0}=\frac{100 n_{\mathrm{D}}}{n_{\mathrm{D}} F_{\mathrm{D}}+n_{\mathrm{M}} F_{\mathrm{M}}}
$$

where $n_{M}$ and $n_{\mathrm{D}}$ are the numbers of moles of MA and DOMA in the mixed monolayer. The HHC dissociation does not influence $\sigma_{0}$ since the dye to matrix molar ratio is very small. The mixture occupies a total area $A$, and
Table I. Comparison of the $\psi_{0} / \sigma_{0}$ Dependence Obtained in the Present Study with Literature Data for Other Systems

\begin{tabular}{cccc}
\hline $\begin{array}{c}\sigma_{0} \text { or } \sigma_{0, \rho}, \\
\mu \mathrm{C} / \mathrm{cm}^{2}\end{array}$ & $\begin{array}{c}\psi_{0} \text { (this study), } \\
\mathrm{mV}\end{array}$ & $\begin{array}{c}\psi_{0} \text { (ref 8), } \\
\mathrm{mV}\end{array}$ & $\begin{array}{c}\psi_{0} \text { (ref 19), } \\
\mathrm{mV}\end{array}$ \\
\hline 2.0 & $25=6$ & 22 & 26 \\
3.8 & $43 \pm 6$ & 37 & 48 \\
7.0 & $67 \pm 6$ & 64 & \\
15.0 & $96 \pm 6$ & 90 &
\end{tabular}

$F_{\mathrm{M}}$ and $F_{\mathrm{D}}$ are the partial molar areas of MA and DOMA, respectively, defined by the equations

$$
\begin{aligned}
& F_{\mathrm{M}}=\left(\frac{\partial A}{\partial n_{\mathrm{M}}}\right)_{\pi, T, n_{\mathrm{D}}}=\left(\frac{\partial F}{\partial X_{\mathrm{M}}}\right)_{\pi, T, n_{\mathrm{D}}} \\
& F_{\mathrm{D}}=\left(\frac{\partial A}{\partial n_{\mathrm{D}}}\right)_{\pi, T, n_{\mathrm{M}}}=\left(\frac{\partial F}{\partial X_{\mathrm{D}}}\right)_{\pi, T, n_{\mathrm{M}}}
\end{aligned}
$$

$F_{\mathrm{M}}$ and $F_{\mathrm{D}}$ are determined graphically from the $F / X_{\mathrm{D}}$ dependence (Figure 8) by extrapolation of the corresponding tangents to $X_{\mathrm{D}}=0$ and $X_{\mathrm{D}}=1$.

The values of $\psi_{0}$ and $\sigma_{0}$ calculated from eqs $3-5$ by using the $\mathrm{p} K_{\mathrm{i}}^{0}, \mathrm{p} K_{\mathrm{i}}$, and the $F / X_{\mathrm{D}}$ data obtained in this study are presented with circles in Figure 9 . The potential values given with triangles are found by HHC titration under monochromatic excitation at $366 \mathrm{~nm}$. The coincidence of the results suggests that the two excitation techniques are equivalent with respect to the fluorometric titration of $\mathrm{HHC}$.

\section{Discussion}

The surface potential values obtained in this study are compared with results from other investigations in Table I. The data presented in the third column are derived from Figure 3 of the publication of Fromherz and Masters ${ }^{8}$ on fluorometric titration of HHC in mixed eicosyltrimethylammonium bromide/methyl stearate monolayers. By use of Langmuir-Blodgett technique, the monolayers have been transferred onto hydrophobic glass substrates covered additionally with several methyl stearate layers. The fluorescence of the embedded $\mathrm{HHC}$ has been measured at the solid/liquid interface (between the last transferred monolayer and the aqueous subsolution) with the aid of a specially developed technique. ${ }^{7}$

From the coincidence of the potential values at the solid/liquid and the gas/liquid interfaces, a conclusion could be drawn that the role of the external (nonaqueous) phase is insignificant. On one hand, this result is to be expected since the image forces which could cause such a difference have similar values in both systems. On the other hand, the $\mathrm{p} K_{\mathrm{i}}$ values determined by the method Fromherz often depend on hydrophobization and, respectively, on the nature of the solid substrate. ${ }^{18,23}$ In the cases of $\mathrm{HHC}$ embedded in a methyl stearate matrix and then transferred onto siliconized glass, glass covered with cadmium arachidate, and glass covered with eicosylamine, the respective $\mathrm{p} K_{\mathrm{i}}^{0}$ values are $9.6,7.7$, and 5.7

(18) Haase, A. Ph.D. Dissertation, Justus Liebig University, Giessen, $1980, \mathrm{p} 72$.

(19) Tokiwa, F.; Ohki, K. J. Phys. Chem. 1966, 70, 3437.

(20) Funasaki, N. J. Colloid Interface Sci. 1977, 60,54.

(21) Hunter, R. J. In Comprehensive Treatise of Electrochemistry; Bockris, J. O.\&M., Conway, B. E., Yeager, E., Eds.; Plenum: New York, 1980; Vol. 1, p 399.

(22) Adamson, A. W. Physical Chemistry of Surfaces, 4th ed.; Wiley: New York, 1982; $p 164$.

(23) Möbius, D.; Cordroch, W.; Loschek, R.; Chi, L. F.; Dhathathreyan, A.; Vogel, V., in preparation.

(24) The decrease of $I$ in the acidic region and its effect on the $\mathrm{p} K^{0} / \mathrm{pH}$ dependence is the object of a separate investigation, to be published additionally. 
(see ref 18). According to the author, these differences should be related to residual charges on the glass surface remaining after hydrophobization.

Similar effects are reported in ref 23 . For the same solid substrates, considerable shifts of the protonation equilibriums have been observed with deposited monolayers containing an amphiphilic porphyrin as well as with deposited $\mathrm{HHC}$ /arachidic acid mixtures. Later model experiments with inversion of the direction of the dipoles in the hydrophobizing monolayer have shown directly the important role of the polarity of the external nonaqueous phase.

In this connection, it is interesting to note that both the $\mathrm{p} K_{\mathrm{i}}{ }^{0}$ and $\mathrm{p} K_{\mathrm{i}}$ values for the compared solid/liquid and the gas/liquid interfaces differ systematically by 0.4 unit while the differences $\mathrm{p} K_{\mathrm{i}}{ }^{0}-\mathrm{p} K_{\mathrm{i}}$ determining the electrostatic potentials shown in Table I are the same. Therefore, when the same but uncharged interface is taken as a reference, the effect of the external phase on $\psi_{0}$ is eliminated, and the potential thus obtained is related only to the monolayer/water charge density in the sense of the Gouy-Chapman consideration.

The data in the fourth column of Table I are recalculated from the results of a potentiometric titration of a micellar solution of $\mathrm{C}_{12} \mathrm{H}_{35} \mathrm{H}\left(\mathrm{CH}_{3}\right)_{2} \mathrm{O}$, performed in ref 19. They refer to the potential established by the positive charges of the aminooxide groups on the surface of the micelle as a result of the ionic equilibrium:

$$
\mathrm{C}_{12} \mathrm{H}_{35} \mathrm{~N}\left(\mathrm{CH}_{3}\right)_{2} \mathrm{O}+\mathrm{H}^{+} \rightleftharpoons \mathrm{C}_{12} \mathrm{H}_{35} \mathrm{~N}^{+}\left(\mathrm{CH}_{3}\right)_{2} \mathrm{OH}
$$

Some serious indications prove the reliability of the $\psi_{0}$ data obtained in this investigation:

(1) In accordance with the theory used in ref 19 for the determination of $\psi_{0}$, a unique experimental dependence of $\mathrm{p} K_{\mathrm{i}}$ on the degree of surfactant dissociation (master curve) has been obtained for all studied concentrations above cmc. As could be expected from the DebyeHückel approximation, this dependence is linear at small $\alpha$.

(2) The $\psi_{0}$ values obtained potentiometrically in ref 19 have been used in another independent spectroscopic determination of the dissociation constants $K_{\alpha}$ of $p$-nitrophenol, brombenzene purple, and chlorophenol red solubilized in the same micelles. ${ }^{20}$ In this way, an independence of the intrinsic dissociation constant $K_{\text {in }}$ on $\psi_{0}$ has been obtained, as expected, according to the equation

$$
\mathrm{p} K_{\mathrm{in}}=\mathrm{p} K_{\alpha}+\frac{e \psi_{0}(\alpha)}{2.3 k T}
$$

The surface charge density of the micelle, $\sigma_{0, m}$, can be evaluated from the degree of surfactant dissociation in the micelle $\alpha_{\mathrm{m}}$, the micelle radius $R$, and the degree of the aggregation of the surfactant $n$, also reported in ref 19:

$$
\sigma_{0, \mathrm{~m}}=\frac{n \alpha_{\mathrm{m}}}{4 \pi R^{2}}
$$

The experimental relationship $\sigma_{0, m} / \psi_{0, m}$ thus obtained (taken up to $50 \mathrm{mV}$ where $R$ can be considered constant $^{19}$ ) coincides within about $10 \%$ with the theoretical dependence for a spherical double layer: ${ }^{21}$

$$
\sigma_{0, \mathrm{~m}}=\frac{2 n_{0} z e}{\chi}\left(2 \operatorname{sh} \frac{z e \psi_{0, \mathrm{~m}}}{2 k T}+\frac{4 \operatorname{th} \frac{z e \psi_{0, \mathrm{~m}}}{4 k T}}{\chi R}\right)
$$

Here $n_{0}$ and $\chi$ are the number ionic concentration and the reciprocal Debye length.

The second term in eq 8 represents the difference between a flat and a spherical interface. Thus one can convert the $\psi_{0, m} / \sigma_{0, m}$ data to $\psi_{0, m} / \sigma_{0, e}$ data, where $\sigma_{0, e}$ is the charge density of an electrically equivalent flat interface having the same surface potential as the micelle:

$$
\frac{\sigma_{0, \underline{m}}}{\sigma_{0, e}}=1+\frac{2}{\chi R\left(\operatorname{ch} \frac{z e \psi_{0, \mathrm{~m}}}{2 k T}+1\right)}
$$

The latter relationship, $\psi_{0, \mathrm{~m}} / \sigma_{0, \mathrm{e}}$, is shown in the fourth column of Table $I$. The coincidence of this date with the results obtained in ref 8 and this study supports the reliability of the fluorometric method.

The determination of the potentials at the gas/liquid interface performed in the present investigation is free of the complications with the solid substrates discussed above. Another advantage is the direct determination of the surface charge density, while in the technique of Fromherz additional measurements of the transfer coefficients are required. Nevertheless, in both cases a common uncertainty of the fluorometric method should be kept in mind by the interpretation of the results. The unknown position of the dye chromophore with respect to the plane of surface charge does not allow one to state a priori if $\psi_{0}$ or $\psi_{\delta}$ is determined. (In our system, $\delta$ is the distance between the $\mathrm{N}$ atoms of DOMA, giving rise to the surface charge, and the $\mathrm{O}$ atom of the dissociating hydroxyl group of the HHC chromophore, regulating its fluorescence.) Since the NMR technique that is often applied to solve this problem in micellar solutions ${ }^{11,18}$ cannot be used here, some indirect indications will be considered below:

(1) The three systems compared in Table I have charged functional groups of different structure, and their surfaces of charge should be differently located. The coincidence of the corresponding potentials observed suggests that the potential of the plane of surface charge is determined in all cases. The alternative explanation, that these are $\psi_{\delta}$ potentials at $\delta$ being equal for all the systems compared, does not seem plausible.

(2) As a result of the potentiometric titration (ref 19), $\psi_{0}$ has been obtained. The coincidence of the values recalculated for an equivalent flat interface with the fluorometric data from this study and ref 8 suggests that the same magnitude is determined in the latter cases.

(3) Taking into account the large dimensions of the chromophore of $\mathrm{HHC}$, it could be expected that in the condensed state of the monolayer matrix at $30 \mathrm{dyn} / \mathrm{cm}$ the latter can be partially or completely squeezed out of the plane of the charged DOMA head groups. If this effect takes place, the measured potentials should be smaller than $\psi_{0}$. This difference should increase in more condensed mixtures, i.e., at smaller $X_{\mathrm{DOMA}}$, and respectively smaller $\sigma_{0}$. However, the comparison of the experimental data obtained in this study and in ref 8 with the Gouy-Chapman $\psi_{0}{ }^{\mathrm{aC}} / \sigma_{0}$ relationship demonstrates the opposite tendency. In both cases, the coincidence between $\psi_{\text {exp }}$ and $\psi_{0}{ }^{G C}$ becomes better when the surface charge density decreases (see Figure 9 of this study and Figure 3 of ref 8 ).

The above arguments give indirect support to the assumption that $\psi_{0}$ is obtained in the present investigation. To prove this in an explicit way, involvement of other proper techniques, justifing the chromophore location, is necessary. 
Acknowledgment. This investigation has been performed in the Max-Planck Institut für biophysikalische Chemie, Göttingen, during the stay of Dr. J. G. Petrov there as a foreign research fellow. He is very apreciative to the Alexander von Humboldt-Stiftung for the award of this fellowship, which made possible the above investigation.

Registry No, 4-Heptadecyl-7-hydroxycoumarin, 26038-835; methyl arachidate, 1120-28-1; dimethyldioctylammonium bromide, 3700-67-2.

\title{
Differential Reflectance Spectroscopy and SERS of Mildly Roughened Silver Electrodes
}

\author{
Mark A. Bryant and Jeanne E. Pemberton* \\ Department of Chemistry, University of Arizona, Tucson, Arizona 85721 \\ Received May 16, 1989. In Final Form: November 6, 1989
}

\begin{abstract}
Differential reflectance spectroscopy has been used to measure the reflectivity of electrochemically roughened $\mathrm{Ag}$ electrodes in $0.1 \mathrm{M} \mathrm{KCl}$ and $0.1 \mathrm{M} \mathrm{KCl} / 0.05 \mathrm{M}$ pyridine. The electrodes were subjected to varying anodic current densities in double-potential-step oxidation-reduction cycles. A correlation exists in both systems between SERS intensities for both $\nu(\mathrm{Ag}-\mathrm{Cl})$ and pyridine ring breathing vibrations and the magnitude of the decrease in reflectivity. Surfaces that exhibit the greatest decrease in reflectivity exhibit the greatest SERS intensity. Scanning electron microscopy is used to characterize surface morphology. The reflectivity spectra are interpreted in terms of absorption by large-scale Ag roughness features. The correlation between decrease in reflectivity and increased SERS intensity is proposed to be due to electromagnetic enhancement effects.
\end{abstract}

\section{Introduction}

Surface-enhanced Raman scattering (SERS) is an analytical technique that is able to provide selective vibrational information about adsorbates within the electrochemical double layer and at the metal-gas interface. In the electrochemical envirunment, oxidation-reduction cycles (ORCs) are the mosi common roughening pretreatment of $\mathrm{Ag}, \mathrm{Cu}$, and $\mathrm{Au}$ slectrodes for SERS. ${ }^{1}$ Despite enhancements of $10^{6}$ that are commonly achieved for adsorbates at Ag electrodes, ORC pretreatment is fraught with irreproducibility and is not well understood. ${ }^{2,3}$ Thus, investigations of ORC procedures are necessary for producing SERS-active surfaces that provide optimum, reproducible Raman enhancements for extensive analytical use.

Previous papers from this research group have shown relationships between SERS intensities and various parameters of the ORC, such as oxidation rate, solution $\mathrm{pH}$, and adsorbate. Tuschel, Pemberton, and Cook reported that the SERS intensity of the $\nu(\mathrm{Ag}-\mathrm{Cl})$ band at $\mathrm{Ag}$ roughened in $1 \mathrm{M} \mathrm{NaCl}$ increased as the anodic current of the ORC increased. ${ }^{4}$ Scanning electron micrographs (SEMs) showed that the $\mathrm{Ag}$ roughness features produced in doublepotential-step ORCs decreased in size as the rate of oxidation increased. The greatest SERS intensity was observed from a surface prepared with an oxidation rate of $18 \mathrm{~mA} / \mathrm{cm}^{2}$. Similarly, Cross and Pemberton reported an optimum SERS intensity for pyridine bands at $\mathrm{Ag}$ electrodes roughened in $0.1 \mathrm{M} \mathrm{KCl} / 0.05 \mathrm{M}$ pyridine with

* Author to whom correspondence should be addressed.

(1) Chang, R. K. Ber. Bunsen-Ges. Phys. Chem. 1987, 91, 296

(2) Tuschel, D. D.; Pemberton, J. E. Langmuir 1988, 4, 58.

(3) Sobocinski, R. L.; Pemberton, J. E. Langmuir 1988, 4, 836. 380 .

(4) Tuschel, D. D.; Pemberton, J. E.; Cook, J. E. Langmuir 1986, 2, an anodic current density of $4 \mathrm{~mA} / \mathrm{cm}^{2}{ }^{5}$ The $\mathrm{Ag}$ roughness feature size was also found to decrease with increasing ORC current density in the $0.1 \mathrm{M} \mathrm{KCl} / 0.05 \mathrm{M}$ pyridine media.

Ag roughness features are important in SERS, because the magnitude of the enhanced electric field giving rise to the enhanced Raman intensities is a function of the size and shape of these roughness features. $1,6,7$ These parameters have been used by many researchers to calculate theoretical enhancements using various electromagnetic (EM) models. ${ }^{2-11}$ For example, Wang and Kerker report that SERS intensities are directly related to the absorption of light by 5-nm-diameter Ag spheres. ${ }^{12}$ These researchers found that this enhancement is dependent on the surface morphology and not the nature of the adsorbate. Absorption of light by these surfaces is also important for charge-transfer (CT) enhancement of SERS. Atomic-scale roughness features of $\mathrm{Ag}$ are thought to be responsible for this type of enhancement. The size of these atomic-scale features is estimated to be no bigger than five or six Ag atoms by Moskovits. ${ }^{6}$ The Ag-adatom complex proposed by Roy and Furtak to be responsible for $\mathrm{CT}$ enhancement in $\mathrm{KCl} /$ pyridine systems is composed of a pyridine molecule bonded to a tetramer of $\mathrm{Ag}$ atoms,

(5) Cross, N. A.; Pemberton, J. E. J. Electroanal. Chem. 1987, 217,

(6) Moskovits, M. Reu. Mod. Phys. 1985, 57, 783.

(7) Otto, A. In Light Scattering in Solids IV; Cardona, M., Güntherodt G., Eds.; Springer-Verlag: Berlin, 1984; $p 289$.

(8) Adrian, F. J. Chem. Phys. Lett. 1981, 78, 45.

(9) Aroca, R.; Martin, F. J. Raman Spectrosc. 1985, 16, 156.

(10) Cline, M. P.; Barber, P. W.; Chang, R. K. J. Opt. Soc. Am. B 1986, $3,15$.

(11) Bloemer, M. J.; Ferrell, T. L.; Buncick, M. C.; Warmack, R. J. Phys. Rev. B 1988, 37, 8015.

(12) Wang, D.-S.; Kerker, M. Phys. Rev, B 1981, 24, 1777. 